\title{
Moving the body to improve oral health
}

\section{Associations between self-reported physical activity and oral health: a cross-sectional analysis in 17,777 Spanish adults. Br Dent J 2020; 228: 361-365. https://doi.org/10.1038/s41415-020-1306-3}

Oral health is multifaceted. Hence it is only reasonable for there to be a complex interplay of numerous lifestyle behaviours to account for when taking a holistic, patient-centred approach to addressing poor oral health. Physical activity being beneficial for the body is already common knowledge. Given how the dental profession are striving to 'put the mouth back into the body', the understudied association between oral health and physical activity warrants further investigation.

This study used data from the 2017 Spanish National Health Survey to examine the relationship between physical activity and the parameters of oral health. Sampling resulted in 17,777 adults aged between 15 and 69 years who had completed the validated IPAQ (International Physical Activity Questionnaire) measuring physical activity levels. As for evaluating oral health, trained interviewers collected self-reported oral health outcomes through conducting interviews with selected participants, during which eight variables were examined through yes-no questions.

Participation in physical activity was found to be favourably associated with some but not all self-reported oral health correlates. Dental caries, dental extraction, gingival bleeding, tooth movement, and missing teeth were all variables significantly less frequent in those who undertook sufficient physical activity. This could be attributed to the favourable inflammatory profile linked to physical activity that plays a role in pathogenesis of oral disease. After adjustment for confounding factors such as age, obesity, smoking status and alcohol consumption, self-reported physical activity was demonstrated to be protective against dental caries, gingival bleeding, tooth movement and missing teeth.

However, this study found that the risk of presence of dental materials was higher in physically active participants. This might be due to tooth erosion or caries due to higher consumption of sports drinks. Alternatively, those with high levels of physical activity may be more health-conscious and thus more likely to receive treatments involving placement of dental materials.

The authors acknowledge that the cross-sectional nature of the present study excludes the possibility of determining the direction of associations, whether positive or negative, and that these may very well be bidirectional. Physical activity potentially aids in preventing various oral complications. But despite growing research highlighting the oral-systemic link, how the majority of lifestyle behaviours are specifically implicated in oral health lacks indisputable evidence. As well as consideration of wealth as a potential covariate, using more objective measures to mitigate potential self-reported bias may provide more certainty as to the relationship with physical activity.

Ji-Yun Stephanie Yeung, Barts and The London



The literature addressing the association between oral health and physical performance is littered with anecdotal evidence suggesting an association between the two. Self-reported studies undertaken at the London 2012 Olympic Games ${ }^{1}$ would suggest that there is a link between poor oral health and athletic performance within elite athletes. A total of 302 athletes from 25 sports were recruited; more than $40 \%$ of them were 'bothered' by their oral health with $28 \%$ reporting an impact on quality of life and performance. Further studies undertaken in professional football in the $\mathrm{UK}^{2}$ and a systematic review ${ }^{3}$ confirmed these suspicions but without any significant proof. The first large representative sample study of oral health in athletes from different sports at elite level was undertaken by Gallagher et al., (2019). This provides robust evidence of the prevalence of oral health diseases and associated performance impacts in elite athletes. ${ }^{4}$

Although experience of oral disease differs by sport, the prevalence, in UK elite and professional athletes, is substantial, with common self-reported performance impacts. Despite reporting positive oral health-related behaviours when screening is undertaken, athletes have substantial amounts of oral disease. Athletes are willing to consider behaviour change related to daily plaque removal, increased fluoride availability and regular dental visits to improve oral health. ${ }^{5}$

There is also the infographic which includes data stating: 'Oral health is poor (dental caries, dental erosion and periodontal diseases are common) in elite athletes and is consistently associated with selfreported performance impacts as shown across studies involving more than 800 athletes.', ${ }^{1,4}$

Why should we be concerned? At the elite level of sport the difference between success and coming second can often be a fraction of a second, a few millimetres or being able to keep going during endurance events. The oral health of elite athletes needs to be consistent with the rest of their general health and wellbeing, to achieve the optimum performance. Missing training and competition due to oral health issues will result in a sub-optimum performance, disappointment and failure at the highest level. Dental screening of elite athletes is proving to be helpful in diagnosing dental disease, and treating/preventing it.

\section{Reference}

1. Needleman I, Ashley P, Petrie A et al. Oral health and impact on performance of athletes participating in the London 2012 Olympic Games: a cross-sectional study. Br J Sports Med 2013; 47: 1054-1058.

2. Needleman I, Ashley P, Meehan L et al. Poor oral health including active caries in 187 UK professional male football players: clinical dental examination performed by dentists. $\mathrm{Br} J$ of Sports Med 2016; 50: 41-44.

3. Ashley P, Di lorio A, Cole E, Tanday T, Needleman I. Oral health of elite athletes and association with performance: a systematic review. Br J Sports Med 2015; 49: 14-19.

4. Gallagher, J, Ashley P, Petrie A, Needleman I. Oral health and performance impacts in elite and professional athletes. Community Dent Oral Epidemiol 2018 46: 563-568.

5. Gallagher, J, Ashley P, Petrie A, Needleman I. Oral health-related behaviours reported by elite and professional athletes. Br Dent J 2019; 227: 276-280. 